\title{
Cerebral Artery Flow Velocity Acceleration and Deceleration Characteristics in Newborn Infants ${ }^{1}$
}

\author{
TONSE N. K. RAJU AND SHIN Y. KIM \\ Division of Neonatology, Department of Pediatrics, University of Illinois College of Medicine at Chicago, \\ Chicago. Illinois 60612
}

\begin{abstract}
To evaluate maturational differences in the cerebral artery blood flow velocity characteristics, we measured the middle cerebral (MCA) and basilar artery blood flow velocity and time intervals using a real-time imaging pulsed Doppler system in 39 preterm and 25 term infants. Preterm MCA and basilar artery mean Doppler spectral area under the curve were $\approx 22$ and $25 \%$ lower than the corresponding term values, with a significant increase during gestation as revealed by regression models. Paradoxically, the mean MCA systolic acceleration and deceleration slopes were $\approx 28$ and $18 \%$ higher in preterm than in term infants: acceleration $701.2 \pm 160 \mathrm{~cm} / \mathrm{s}^{2}$ versus $546.4 \pm 156.5 / \mathrm{s}^{2}(p<0.005)$, and deceleration $222.2 \pm$ $71.6 \mathrm{~cm} / \mathrm{s}^{2}$ versus $188.2 \pm 52.2 \mathrm{~cm} / \mathrm{s}^{2}(p<0.05)$, respectively. Similarly, acceleration and deceleration pressure half times for MCA were about 15 and $18 \%$ shorter in the preterm $(p<0.01$ and $<0.005)$. Multiple regression models controlling for velocity and frequency terms revealed an inverse relationship between gestational age and MCA acceleration $(p<0.005)$, and a positive relationship between gestational age and MCA acceleration pressure half times $(p<0.02)$. In preterm infants the MCA specific index of pulsatility was about $32 \%$ higher $(p<0.05)$ than in the term, which was also inversely related to gestational age in a regression model controlling for velocity terms $(p$ $<0.02$ ). However, the basilar artery acceleration and deceleration slopes were not significantly different between term and preterm groups, nor showed a significant gestational age effect in the multiple regression models. The results indicate that despite a lower volume flow, the rate of rise of MCA velocity is much higher in preterm than in the term infants; during maturation the velocity waveforms assume a smoother contour. These hemodynamic characteristics could in part reflect immature vessel wall viscoelastic properties, particularly lack of elastin which could result in a reduced windkessel phenomenon. (Pediatr Res 26: 588-592, 1989)
\end{abstract}

\section{Abbreviations}

MCA, middle cerebral artery

BA, basilar artery

$V_{\text {mon }}$, mean Doppler spectral velocity

$V_{\text {ed }}$, end-diastolic velocity

AUC, area under the Doppler spectral curve

APHT, acceleration pressure half time

DPHT, deceleration pressure half time

Received February 27, 1989; accepted July 14, 1989

Correspondence T. N. K. Raju, Department of Pediatrics, (m/c 856), $840 \mathrm{~S}$. Wood St. Chicago, IL 60612.

Supporied in part by grants from the National Institutes of Health Grant SIO RRO 2528-01 and Alaska Neonatal/Perinatal Associates, Anchorage, Alaska.

' Presented in part at the 1989 annual meeting of the Society for Pediatric Research, Washington, DC.

\author{
SIP, specific index of pulsatility \\ AT, acceleration time \\ DT, deceleration time \\ BP, blood pressure
}

Advances in Doppler and ultrasound technology permit quantitation of blood flow velocity and evaluation of flow changes in regional arteries of newborn infants having a variety of clinical disorders (1-5). Although pulsed Doppler systems measure velocity in real units, they do not provide direct volume flow information, therefore the AUC has been used as one of the measures to indirectly estimate volume flow $(6-8)$.

Cerebral vessel histology and maturation studies (9-11) suggest that in preterm gestations, even the major intracranial arteries lack collagen, smooth muscle, and elastic elements, and the maturational pattern may not be uniform (11). Because vessel distensibility characteristics that influence waveform shapes also affect microcirculatory flow, we asked whether any differences exist in the velocity waveforms among newborn infants of varying gestation. We had previously noted that premature infants' cranial artery pulsed Doppler velocity waveforms were more acute, having a steep rise followed by a rapid drop (12).

The purpose of this study was therefore to examine the effect of gestational age on cerebral velocity waveform characteristics and their possible regional differences. We adapted well established Doppler echocardiographic methods that are known to reflect both left ventricular hemodynamic status as well as aortic wall properties (13-17).

\section{MATERIALS AND METHODS}

Subjects. The studies were approved by the institutional review board and parent's consent was obtained. Healthy term newborn infants free of obvious malformations were enrolled into the study if they met the following criteria: the 5-min Apgar score $>7$; no cardiopulmonary or neurologic disorders; no clinical evidence of patent ductus arteriosus; on no medications, and normal cranial ultrasound findings. Healthy preterm infants who were not receiving anticonvulsants or theophylline, and not having ultrasound evidence of intracranial hemorrhage or periventricular leukomalacia were eligible, as were those who had fully recovered from mild respiratory distress (of less than 6 days) but were not currently on assisted ventilation or supplemental oxygen. Inasmuch as these were well babies, blood gas tensions were not measured. The subject selection was not randomized. Infants were asleep (clinical), and in prone recumbent position during the Doppler study.

Doppler methods. The technique of measuring regional cerebral blood flow velocities using the commercial phased array sector scanner (Hewlett Packard 77030A, Palo Alto, CA) along with a $5.0 \mathrm{mHz}$ short focus transducer has been previously 
reported $(4,5)$. A transcranial view was used to study the MCA $0.5 \mathrm{~cm}$ lateral to its origin, and a fontanellar view to study the $\mathrm{BA}$ in front of the pons. The sample volume was $0.5^{3} \mathrm{~mm}$. The blood flow/Doppler incident angle (measured with the electronic calipers) was always between 0 and $10^{\circ}$, so that velocities measured were accurate to within $3 \%$ (13); hence no angle correction was done.

At least $1 \mathrm{~min}$ of consistent velocity display was videotaped for later analysis. Using built-in electronic calipers and computer package four or five consecutive Doppler waveforms were analyzed to measure all of the following Doppler variables (Fig. 1): $V_{\max }, V_{e d}$, by integrating the waveform over a cardiac cycle, the time averaged $V_{m n}(13,18)$; velocity acceleration slope $\left(\frac{\Delta V}{\Delta t}\right.$, where $t$ is the time to $V_{\max }$, or AT); late systolic deceleration slope $\left(\frac{\Delta v}{\Delta t}\right.$, where $t$ is DT); and APHT and DPHT (as per the modified Bernoulli equation; the time between $V_{\max }$ and the point on ascending/descending Doppler wave segment that is equal to $V_{\max }$ divided by $\sqrt{2}$, or $\left.\approx 1.4\right)(13,14,19)$. Using velocity data, we computed the specific index of pulsatility (SIP $=\frac{V_{m n}}{V_{e d}}$ 1) (see "Appendix").

Doppler calculations were done by placing the electronic caliper cross-hair at specified points on the waveforms (Fig. 1), identifying the leading edge of spectral display and the steepest parts of ascending and descending waveform segments $(13,14$, 19). Inasmuch as the dicrotic notch is not clearly evident in the peripheral flow velocity curves, end-systole was identified as the point on the deceleration segment coinciding with a steady forward flow. The measurements were precise to $\pm 0.167 \mathrm{~cm}$ for velocity, and $\pm 0.005 \mathrm{~s}$ for time.
The data were analyzed by two-tailed $t$ test for comparing group mean values, and by multiple regression models with a general formula $\hat{\mathrm{Y}}=\hat{\mathrm{a}}+\hat{\beta}_{1} \times \mathrm{X}_{1}+\hat{\beta}_{2} \times \mathrm{X}_{2} \ldots$ In addition to either gestational or postconceptional age, we included a velocity and a frequency term in the regression models. This method helps isolating the effect of one variable (such as gestational or postconceptional age) after controlling for changes in other variables, and does not violate statistical principles of multiple regression (Gauss Markov conditions). Because multiple models could increase $\alpha$ error, a $p<0.02$ was considered statistically significant, and $p>0.02-<0.05$ as marginally significant as per the modified Bonferroni correction (20). The magnitude of regression coefficients $(\hat{\beta}), \mathrm{SE}_{\hat{\beta}}$, and their statistical significance were noted in those models that were significant.

\section{RESULTS}

Clinical variables. The right MCA was studied in all the 64 cases whose clinical data are given in Table 1. The BA was studied in 53/64; technical difficulties precluded studies in the remaining 11 . Although the mean postnatal age at study was higher in the preterm group, $23 / 39$ were between $1-7 \mathrm{~d}$ of age. No statistically significant difference was noted in the mean hematocrit values (obtained within 12 h of Doppler studies), whereas an expected difference in the mean BP and pulse pres. sure was seen. The mean $( \pm S D)$ insonation gate depth was 3.1 $\pm 0.4 \mathrm{~cm}$ for term MCA; $2.45 \pm 0.44$ for preterm MCA; $6.51 \pm$ $0.52 \mathrm{~cm}$ for term BA; and $5.67 \pm 0.78 \mathrm{~cm}$ for preterm BA.

$V$ elocity and time intervals. The $V_{\max }$ and $V_{m n}$ were similar between term and preterm for both vessels. Preterm MCA mean $V_{\text {ed }}$ and AUC were 25.9 and $17.7 \%$ lower, and preterm BA mean $\mathrm{V}_{\text {ed }}$ and $A U C$ were 26.1 and $25.3 \%$ lower than the respective

A

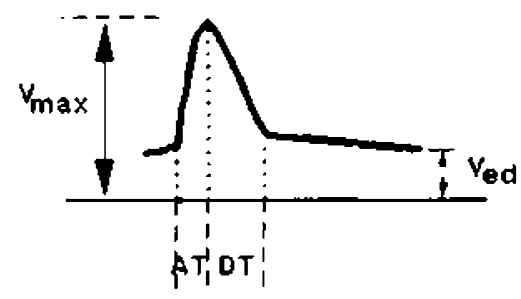

B

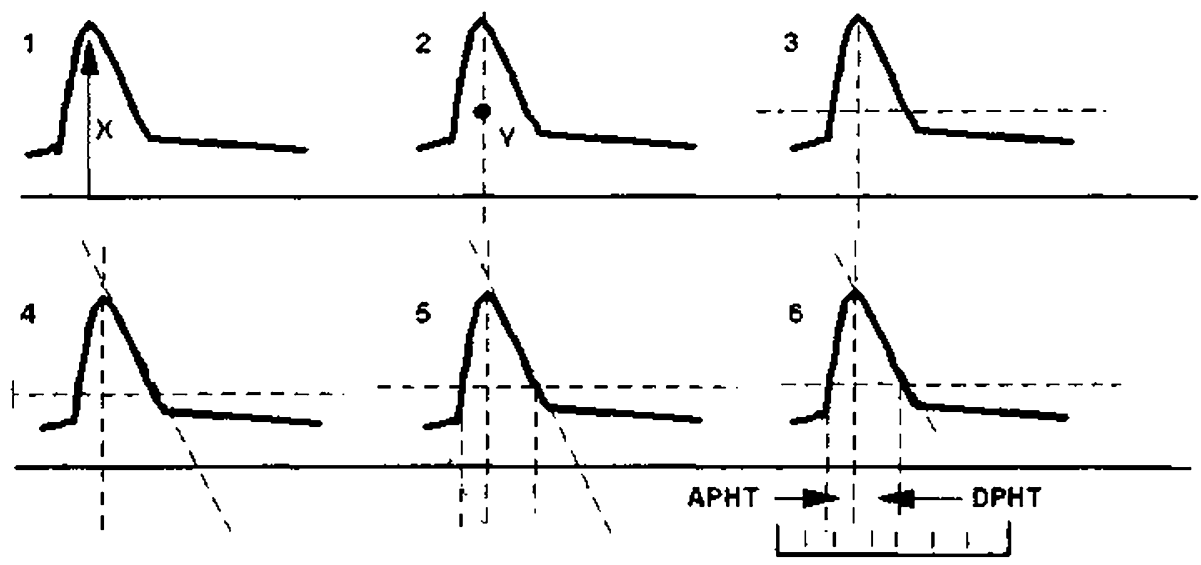

Fig. 1. Doppler calculations. $A$, Doppler measurements were done from the video-taped images; the electronic cross-hair was piaced at specific points, and the calculations were performed by the built-in computer package, interactively. $V_{\max }$ and $V_{e d}$ were calculated as shown. $V_{m n}$ was calculated by tracking the spectral waveform over the entire cardiac cycle. Acceleration slope was obtained by $\Delta$ velocity (between the beginning of waveform uptake to $\mathrm{V}_{\max }$ ) divided by the AT; deceleration slope was obtained by $\Delta$ velocity (between $\mathrm{V}_{\max }$ and end-systole), divided by DT. $B$, Steps in calculating pressure half times: $1, \mathrm{~V}_{\max }$ is shown as $\mathrm{X} .2, \mathrm{~V}_{\max }$ divided by $\sqrt{2}(\approx 1.4)$ provides velocity $(Y)$ at half peak pressure. $3, A$ horizontal line is drawn through Y. 4-6, Acceleration and deceleration pressure half times (in ms) are the time intervals between the point of peak velocity and the points where the horizontal line through $Y$ intercepts the Doppler waves (19). 